\title{
Atendimento de saúde para pessoas idosas vítimas de violência no município do Rio de Janeiro
}

\author{
Health care for elderly victims of violence in Rio de Janeiro
}

Adalgisa Peixoto Ribeiro ${ }^{1}$ Edinilsa Ramos de Souza ${ }^{1}$ Fabiana Castelo Valadares ${ }^{1}$
${ }^{1}$ Centro Latino Americano de Estudos de Violência e Saúde (Claves), Escola Nacional de Saúde Pública (Ensp), Fundação Oswaldo Cruz (Fiocruz). Av. Brasil 4036/ 700, Manguinhos. 21040-361 Rio de Janeiro RJ.

adalpeixoto@yahoo.com.br
Abstract The scope of this article is to chart, define and qualify the healthcare provided by the Brazilian Unified Health System (SUS) and outsourced services to elderly people in situations of violence in Rio de Janeiro, based on major policies aimed at this group. This is an exploratory study that triangulated quantitative and qualitative methods. The introduction and implementation of policies is differentiated between the units: they are more thoroughly implemented at the pre-hospital and hospital level and less at rehabilitation level. The challenges involve the professional's ability to identify, attend and report cases of violence; to include the topic in care of the elderly; to give visibility to the specific requirements for elderly women; to instruct and orient families about care, creating strategies to redeem the bonds of affection; to monitor the changes that lead to functional disability and enhance the quality of life of the elderly.

Key words Health of the elderly, Public policies, Health care
Resumo O presente artigo objetiva mapear, caracterizar e qualificar o atendimento de saúde prestado pelos serviços próprios e conveniados do SUS a idosos em situação de violência no município do Rio de Janeiro, baseando-se nas principais políticas dirigidas a esse grupo. Trata-se de estudo exploratório que triangulou métodos quantitativo e qualitativo. A implantação e a implementação das politicas é diferenciada entre as unidades: são mais cumpridas nas do nível pré-hospitalar e hospitalar e menos nas de reabilitação. Como desafios aos serviços estão: a capacitação dos profissionais para identificar, atender e notificar os casos de violência; incluir o tema na atenção ao idoso; dar visibilidade às especificidades da atenção à mulher idosa; apoiar e orientar as famílias para o cuidado, criando estratégias para resgatar os laços afetivos; vigiar as alterações que levam a incapacidades funcionais; $e$, promover a qualidade de vida do idoso.

Palavras-chave Saúde do idoso, Políticas públicas, Atenção à saúde 


\section{Introdução}

A violência cometida contra os idosos tem tomado grandes proporções na sociedade contemporânea e tem se tornado mais visível devido ao aumento do número deles na população, mas também pelas conquistas de direitos em leis e políticas nacionais e internacionais.

Essa violência, entendida como atos ou omissões que resultem em danos físicos ou psicológicos ao idoso ${ }^{1}$, impacta diretamente o setor saúde que tem sido exigido a responder às demandas por cuidados cada vez mais complexos e especializados para atender ao idoso. Tal impacto pode ser observado através dos indicadores de morbimortalidade disponibilizados pelo Ministério da Saúde. Em 2008, 20.303 idosos morreram por causas externas (acidentes e violência), o que representou 3,1\% das mortes dessas pessoas no Brasil, ocupando a sétima causa de óbitos nessa faixa etária. Nesse mesmo ano, $65,4 \%$ dos mortos por essas causas precisaram de atendimento e faleceram quando ainda estavam sendo atendidos em uma unidade de saúde. No município do Rio de Janeiro 1.364 idosos morreram por causas externas, sendo que $86,1 \%$ desses óbitos aconteceram quando estavam sob os cuidados de um serviço de saúde, em $2008^{2}$.

A violência não letal, mas grave ao ponto de levar o idoso a procurar atendimento, pode ser observada pelos dados de internação hospitalar. Em 2009, as causas violentas perfizeram 6\% das hospitalizações de idosos no país; na capital do Rio de Janeiro representaram 6,8\% do total de idosos internados ${ }^{3}$. Para fazer frente a essa demanda, o tema da violência está incluído em políticas e as leis brasileiras que orientam o atendimento de saúde para idosos, bem como para as vítimas de violência e constituem o embasamento da presente investigação: a Política Nacional de Redução da Morbimortalidade por Acidentes e Violências (PNRMAV) $)^{4}$, o Estatuto do Idoso $^{5}$ e a Política Nacional de Saúde da Pessoa Idosa (PNSPI) ${ }^{6}$.

Essas políticas preconizam, entre outras questões, a promoção do envelhecimento ativo com adoção de comportamentos e ambientes saudáveis; o direito à atenção integral à saúde através do Sistema Único de Saúde (SUS), com ações intra e intersetoriais; a capacitação dos recursos humanos e a orientação dos cuidadores e familiares assegurando a qualidade da atenção oferecida; a obrigação da notificação dos casos suspeitos ou confirmados de violência aos órgãos públicos e as ações de prevenção desses agravos.

A PNRMAV prevê especificamente a monitorização da ocorrência de acidentes e de violên- cias, bem como a ampliação e consolidação do atendimento nos níveis pré-hospitalar, hospitalar e de reabilitação ${ }^{7}$.

Essas políticas estabelecem que toda a rede de saúde deve estar sensibilizada e apta a prestar o atendimento adequado às pessoas vítimas de violência. Partindo dessa premissa, o objetivo do presente artigo é mapear, caracterizar e qualificar o atendimento de saúde prestado pelos serviços próprios e conveniados do SUS a idosos em situação de violência no município do Rio de Janeiro.

\section{Metodologia}

Este trabalho analisa dados de uma pesquisa maior que realizou um diagnóstico de sistemas locais de saúde para atender aos agravos provocados por acidentes e violências contra idosos em cinco capitais brasileiras: Manaus, Recife, Brasília, Rio de Janeiro e Curitiba. A investigação triangulou métodos quantitativo e qualitativo, visando integrar e ampliar as contribuições teórico-metodológicas de cada uma dessas abordagens $^{8}$. O presente artigo realiza um estudo exploratório dos serviços da rede SUS do município do Rio de Janeiro, buscando mapeá-los, caracterizá-los e qualificar o atendimento prestado a idosos vítimas de violência nos níveis pré-hospitalar, hospitalar e de reabilitação. Não foram incluídas na pesquisa as instituições de longa permanência como asilos e abrigos.

O município do Rio de Janeiro foi selecionado nesse trabalho por ser uma capital com elevada proporção de idosos na população $(14,7 \% \mathrm{em}$ $2008)^{9}$ e por registrar elevados percentuais de mortes e internações destes por causas externas em relação ao país e às outras capitais. Além disso, também foi escolhido pela complexidade de sua rede de atenção que comporta estabelecimentos de saúde municipais, estaduais e federais ${ }^{10}$. É importante destacar que, embora esse seja um estudo descritivo da realidade do atendimento de saúde oferecido a pessoas idosas no município do Rio de Janeiro, sua relevância está na identificação de lacunas, fragilidades e possibilidades da rede pública de saúde, podendo subsidiar ações específicas de profissionais e gestores. Além disso, este estudo se justifica pelo pouco conhecimento sobre essa realidade, pela escassez de publicações sobre o tema e por servir como parâmetro para outras redes locais de saúde do Sistema Único que convivem com os mesmos problemas.

A rede de proteção e a garantia de direitos da pessoa idosa no município do Rio de Janeiro é 
composta por uma estrutura de atendimento social, jurídico, de segurança e também de saúde. Entre os órgãos que efetivamente prestam atendimento ao idoso encontram-se as delegacias especializadas; a Defensoria Pública, que tem um núcleo especializado no atendimento à esse grupo; a Vara da Infância Juventude e Idoso; o Ministério Público ${ }^{11}$; o Centro de Referência Especializado de Assistência Social (CREAS); o Centro Especializado de Atenção e Prevenção da Violência contra a Pessoa Idosa, que oferece atenção jurídica, social e psicológica; o Conselho de Direitos; as instituições de longa permanência e os serviços de saúde em seus diversos níveis de complexidade do atendimento. O presente artigo enfoca apenas os serviços da rede de saúde.

O estudo foi realizado em duas fases distintas. A primeira buscou mapear e caracterizar os serviços da rede SUS que atendem a pessoas idosas em situação de violência no município do Rio de Janeiro e a segunda visou qualificar o atendimento oferecido.

$\mathrm{Na}$ primeira fase do estudo usou-se a técnica Bola de Neve, com a realização de quatro entrevistas com gestores das áreas de saúde do idoso, atendimento pré-hospitalar, hospitalar e de reabilitação que indicaram serviços da rede SUS e conveniados que efetivamente realizam atendimento ao idoso no município. A partir dessas informações foram identificados 41 serviços: 24 do nível pré-hospitalar (14 equipes da Estratégia Saúde da Família [ESF], duas unidades de atenção básica, três de atenção básica ampliada, dois Pronto Socorro [PS] atendimento 24 horas, três ambulatórios especializados para acompanhamento de médio e longo prazo), 15 do nível hospitalar (13 gerais e dois de referência); dois serviços de reabilitação (um de nível intermediário e um de referência).

$\mathrm{Na}$ segunda fase, foi selecionada uma amostra de conveniência de unidades de saúde para a qualificação do atendimento, sendo: um serviço pré-hospitalar móvel, uma ESF por Área de Planejamento Sanitário (AP), os serviços especializados na atenção ao idoso, os hospitais e os serviços de reabilitação inicialmente mapeados. Assim, dos 41 serviços descritos na primeira fase do estudo, 29 tiveram seu atendimento qualificado na segunda fase: 12 do nível pré-hospitalar (cinco da ESF, três da Atenção Básica Ampliada, dois PS 24 horas, um ambulatório especializado e uma unidade pré-hospitalar móvel), 15 hospitais e dois serviços de reabilitação.

A essa amostra aplicou-se um questionário construído com questões abertas e fechadas visando qualificar o atendimento oferecido: iden- tificação do serviço, estrutura existente, organização e registro de dados.

Ainda na segunda fase foram realizadas nove entrevistas: quatro com gestores de serviços e cinco com profissionais (médico e socorrista de serviço pré-hospitalar móvel, gerontólogo, médico emergencista e fisioterapeuta). Essas entrevistas semiestruturadas contemplaram o fluxo, a caracterização e as especificidades do atendimento ao idoso; família e redes de proteção e avaliação dos serviços.

As informações dos questionários foram processadas em um banco de dados, no programa CSPro versão 3.2. Após as correções e crítica os bancos foram convertidos para o software SPSS versão 13.0, onde foi realizada a descrição de frequências.

As entrevistas foram transcritas, incluídas em um banco que as agrupou de acordo com os sujeitos e os temas abordados e analisadas com base no referencial de análise de conteúdo adaptado de Bardin ${ }^{12}$ por Minayo ${ }^{13}$.

A coleta de dados ocorreu de dezembro de 2006 a março de 2008, com aprovação do Comitê de Ética em Pesquisa da Escola Nacional de Saúde Pública, da Fundação Oswaldo Cruz e da Secretaria Municipal de Saúde do município.

\section{Resultados e discussão}

\section{Mapeamento e caracterização dos serviços de saúde que atendem idosos vítimas de violência}

Nas entrevistas com os gestores foi unânime a declaração de que no município não existem serviços específicos para atender a idosos vítimas de violência. É possível obter atendimento especializado para a saúde do idoso, mas sem o enfoque nesse tema.

Este fato pode ser compreendido também pelo pequeno número de profissionais com formação em geriatria atuando na rede de saúde do município: 36 geriatras, sendo que 19 deles atendem nos serviços do SUS ${ }^{14}$.

Alguns gestores apontaram dificuldades para essa atenção como a falta de capacitação dos profissionais para identificar e atender os casos, a falta de sensibilização dos próprios gestores e profissionais de saúde e a ausência de uma rede de proteção bem articulada para o encaminhamento correto.

Em relação ao treinamento dos técnicos, um gestor afirmou que os recursos orçamentários, 
em grande parte, são absorvidos pelas emergências hospitalares e por esse motivo, a atenção ao idoso, nos seus diversos âmbitos, ainda não é prioridade na secretaria de saúde do município. $\mathrm{O}$ treinamento para abordagem do idoso e, principalmente, aquele vítima de violência, ainda é um desafio para os gestores cariocas. Minayo e Deslandes ${ }^{10}$ identificaram essa dificuldade no município: entre 22 serviços de saúde pesquisados, apenas em 22,7\% deles a última capacitação oferecida aos profissionais ocorreu há mais de um ano e em $18 \%$ deles a busca por essa formação foi individual.

Solicitados a indicar os serviços de saúde, que mesmo sem qualquer especificidade, atendem a idosos em situação de violência, esses gestores esclareceram que as unidades de emergência são referência no atendimento às vítimas em geral e o idoso é incluído nessa atenção ao trauma. Essa indicação, em parte, está de acordo com as premissas das políticas em que todos os serviços devem estar preparados para abordar as vítimas de violência. No entanto, destoa das leis quando apontam somente as unidades de emergência como aquelas aptas a receber o idoso vitimizado.

Fica claro nos depoimentos dos gestores a associação da violência ao trauma, o que foi também visível no trabalho de Albuquerque ${ }^{15}$ com profissionais da atenção pré-hospitalar móvel. No estudo dessa autora, os profissionais assumiram que foram preparados para atender ao trauma (uma consequência da violência física) e não para abordar a violência em sua complexidade; $25,6 \%$ deles acreditam que para se caracterizar como ato violento é preciso que haja lesão, ferimento ou algum tipo de trauma físico. É importante lembrar que a violência pode se manifestar em diversas formas, além do abuso físico, os idosos também sofrem com os abusos psicológicos, financeiros, abandono, negligências, entre outros ${ }^{1}$.

Para a mulher vítima de violência os gestores indicaram algumas unidades de referência para a atenção à saúde, entre elas, algumas ligadas a maternidades, a hospitais estaduais e também universitários. Esses serviços oferecem atenção direcionada para a mulher, sem levar em conta as especificidades da mulher idosa. Essa maior visibilidade, em geral, se dá porque esse é um grupo que já possui políticas públicas e de inclusão na agenda do setor saúde que é bem anterior ao tema da violência contra o idoso.

Pensando no atendimento aos idosos, os gestores citaram unidades da atenção pré-hospitalar (ESF, Postos de Saúde) que realizam trabalhos com eles, mas sem abordar a questão da violência. Assim, promovem grupos para tratamento da hipertensão, diabetes e oficinas terapêuticas com realização de exercícios físicos. Também indicaram ambulatórios especializados na atenção ao idoso, vinculados à Universidade Estadual.

\section{Qualificação do atendimento de saúde a pessoas idosas vítimas de violência}

$\mathrm{Na}$ qualificação do atendimento oferecido, que corresponde à segunda fase do trabalho, os 29 serviços selecionados (12 pré-hospitalares, 15 hospitalares e dois de reabilitação) estão vinculados ao governo municipal $(79,3 \%)$, estadual $(10,3 \%)$ e federal (10,3\%).

A informação sobre o número de atendimentos realizado no ano de 2006 revela a invisibilidade da violência contra o idoso nas unidades de saúde. Muitas não dispunham das informações referentes a esses atendimentos e, em outras, parece haver um sub-registro dos casos. Talvez isso ocorra pela falta de identificação da violência camuflada por suas consequências, como fraturas, problemas psicológicos, doenças sem causa aparente e recidiva de problemas anteriores. $\mathrm{Na}$ Tabela 1 é possível visualizar o quantitativo do atendimento informado pelos serviços.

\section{Estrutura existente}

As 11 unidades de atendimento pré-hospitalar, excluindo-se a de pré-hospitalar móvel, que

Tabela 1. Número de atendimentos realizados pelos serviços pesquisados, segundo nível do atendimento. Rio de Janeiro, 2006

\begin{tabular}{lccc}
\hline Número de atendimentos & Pré-hospitalar & Hospitalar & Reabilitação \\
\hline Total & 198.860 & 1.370 .198 & 5.083 \\
De idosos & 73.156 & $45.927^{\star}$ & 73 \\
De idosos por acidentes & - & - & $5^{\star *}$ \\
De idosos por violência & - & - & - \\
\hline
\end{tabular}

* apenas 5 hospitais informaram; ** apenas um serviço de reabilitação informou; - sem informação 
participaram da pesquisa contam com sala de recepção em todas elas; sala de arquivo de prontuário e consultório médico em 10 unidades; sala para serviço social em 8 serviços; consultório odontológico em 7; cadeira de rodas, farmácia e sala para curativos contaminados estão presentes em 6 unidades. Esses resultados estão em conformidade com a Portaria GM 204816, que classifica esses itens como obrigatórios para os serviços não hospitalares de atendimento a urgência e emergência, considerando-se que aqui também estão incluídas 6 unidades desse tipo. Outros itens da estrutura física que, apesar de obrigatórios para esse nível da atenção, foram encontrados em poucas unidades eram laboratório de patologia clínica em 5 delas; laboratório de radiologia, sala de sutura e de gesso em 4; sala de triagem do risco, de inaloterapia/medicação em 3 serviços; e por fim, as salas de observação de pacientes até 8 horas, de reanimação e estabilização (urgência) e acesso diferenciado para ambulâncias aparecem em somente 2 dos serviços préhospitalares pesquisados.

Onze hospitais informaram que possuem 1.861 leitos de adulto, sendo 8,1\% deles de UTI. Essa proporção de leitos de UTI está dentro do preconizado ( $4 \%$ a $10 \%$ em relação ao número total de leitos) para as unidades hospitalares ${ }^{16}$. No entanto, os profissionais dos serviços hospitalares pesquisados atribuíram notas baixas na avaliação dos leitos/vagas em sua própria unidade $(5,7)$, para as instalações físicas $(5,4)$ e para as vagas em outros serviços $(5,0)$. A falta de vagas para a grande demanda nesses serviços revela alguns nós para a gestão do atendimento hospitalar como a lenta rotatividade dos leitos, a insuficiência de recursos humanos e a falta de leitos de retaguarda ${ }^{17}$. Outra questão que dificulta a disponibilidade de vagas nos hospitais é o fato de que pacientes vítimas de violência necessitam de uma internação mais longa ${ }^{18}$ e quando se trata de pessoa idosa, vítima de violência, essa ocupação do leito se torna ainda mais prolongada.

Em todos os hospitalais pesquisados existem equipamentos para a realização de eletrocardiografia e os recursos para análise clínica e laboratorial. Entretanto, os equipamentos para angiografia, cirurgia coloproctológica e ressonância nuclear magnética são encontrados em poucos hospitais, revelando uma necessidade de investimento nessas instituições (Tabela 2). Os dados sobre a estrutura existente ficaram comprometidos, pois não foram informados em alguns hospitais.

Os depoimentos indicaram que os equipamentos existem, mas nem sempre estão disponí-
Tabela 2. Distribuição do número de serviços hospitalares pesquisados segundo itens da estrutura

\begin{tabular}{|c|c|c|}
\hline \multirow{2}{*}{ Recursos } & \multicolumn{2}{|c|}{ Hospitalar } \\
\hline & $\mathrm{N}=15$ & Sem inf. \\
\hline Eletrocardiografia & 15 & - \\
\hline Análise clínica/ laboratorial & 14 & 1 \\
\hline Radiologia Convencional & 14 & 1 \\
\hline Endoscopia & 12 & - \\
\hline Ultrassonografia & 12 & 1 \\
\hline Cirurgia ortopédica e traumatológica & 12 & 1 \\
\hline Cirurgia geral & 12 & 1 \\
\hline Ecocardiografia & 10 & 2 \\
\hline Tomografia computadorizada & 9 & 3 \\
\hline Cirurgia ginecológica & 9 & 2 \\
\hline Neurocirurgia & 7 & 4 \\
\hline Banco de sangue & 6 & 6 \\
\hline Cirurgia cardiovascular & 5 & 3 \\
\hline Intensificador de imagem & 4 & 6 \\
\hline Hemodinâmica & 4 & 5 \\
\hline Broncoscopia & 4 & 5 \\
\hline Banco de tecidos & 3 & 5 \\
\hline Angiografia & 2 & 5 \\
\hline Cirurgia coloproctológica & 2 & 6 \\
\hline Ressonância nuclear magnética & 1 & 4 \\
\hline
\end{tabular}

veis ou em bom estado de conservação para o caso de uma emergência, retardando o atendimento, como descreve um técnico: [...] chegando no hospital não tinha tomografia, ai eu fui para o hospital $X$, tinha tomografia, mas não tinha o neuro, eu fui parar no Hospital Y com a senhora. O socorro começou as 19 horas e o término foi a uma e pouca da manhã.

Os recursos de radiologia convencional estão presentes nas duas unidades de reabilitação, os de análise clínica laboratorial, equipamentos para urodinâmica, ultrassonografia, audiometria e medicina nuclear não são encontrados em apenas um serviço. Entretanto, essas unidades se articulam com outros serviços para esse suporte laboratorial.

Os dois serviços de reabilitação pesquisados realizam avaliação da indicação, prescrição, treinamento, dispensação e acompanhamento dos pacientes que precisam usar órteses. Em relação às próteses, somente um serviço informou que avalia, prescreve e dispensa esses recursos. Os meios auxiliares de locomoção como bengalas, andadores e outros têm sua indicação, prescrição e treinamento nos dois serviços de reabilitação, mas sua dispensação ocorre em apenas uma das unidades estudadas. O fornecimento desses 
recursos às vítimas de violência é garantido pela $\mathrm{PNRMAV}^{4}$, mas no município do Rio de Janeiro esses pacientes encontram dificuldades para obter as próteses e os meios auxiliares de locomoção nas unidades de reabilitação.

Dentre todos os serviços pesquisados, nos três níveis da atenção, $65,5 \%$ possuem meios próprios para a transferência de pacientes e $17,2 \%$ se articulam com outros serviços para esse transporte, como recomendado pelas políticas ${ }^{16}$.

Duas unidades pré-hospitalares e seis hospitais possuem protocolos para atender idosos em situação de violência. Nenhum dos serviços de reabilitação conta com esse recurso que é preconizado pela PNRMAV ${ }^{4}$ visando orientar essa atenção em normas específicas para o tratamento das vítimas, mostrando que esse tema parece não ter sido inserido plenamente no atendimento de saúde.

No quadro de profissionais de nível superior destacam-se enfermeiros, médicos clínico e ortopedista. Por outro lado, parece existir uma deficiência de gastroenterologistas, coloproctologistas e cirurgiões cardiovascular e torácico. Do total de médicos (1.207) informados pelos serviços, $1,6 \%$ (20) são geriatras, percentual maior que o informado pelo Datasus para o ano da pesquisa (2007) que foi de $0,2 \%$ do total de médicos disponíveis na rede de saúde do municí$\mathrm{pio}^{14}$. Talvez isso esteja indicando que os serviços abordados são aqueles que concretamente mais atendem idosos no Rio de Janeiro, mas ainda é um percentual pequeno de especialistas para fazer frente a uma demanda crescente. Entre os profissionais de nível técnico destacam-se os técnicos/auxiliares de enfermagem que aparecem em número expressivo (Tabela 3).

A autoavaliação dos serviços, realizada através de notas atribuídas pelos respondentes, aponta que o número de profissionais para o atendimento a idosos vítimas de violência é relativamente adequado, com média superior a 6 (préhospitalar 7,2, hospitalar 6,6 e reabilitação 9,0). Entretanto, nas entrevistas destacam-se as avaliações que relacionam as deficiências no atendimento à insuficiência quantitativa da equipe disponível nas unidades pesquisadas. Um exemplo disso é o número de geriatras citados na Tabela 3 que não contempla as diretrizes das políticas relacionadas à saúde do idoso sobre a presença de pelo menos um profissional com essa especialidade em cada serviço.

Dentre os serviços pesquisados $44,8 \%$ informaram ter em seu quadro profissionais com formação em gerontologia. As categorias citadas foram médico, assistente social, psicólogo, en- fermeiro, fonoaudiólogo, nutricionista, fisioterapeuta e odontólogo, totalizando 68 profissionais: 31 atuando no nível pré-hospitalar, 35 no hospitalar e 2 no de reabilitação.

Apenas 31\% das unidades estudadas possuem técnicos capacitados para identificar os casos de violência contra idosos, 34,5\% têm profissionais capacitados para atender a esses casos e $37,9 \%$ contam com pessoas preparadas para registrar sistematicamente a informação. Essa formação para lidar com os casos de violência vem sendo pesquisada por alguns autores que apontam as lacunas ainda existentes sobre esse tema na graduação de profissionais de saúde e também na capacitação continuada ${ }^{17,19,20}$. Esse item é fonte de preocupação de alguns gestores, como já citado anteriormente.

\section{Organização dos serviços para $o$ atendimento}

Os pacientes chegam às unidades pesquisadas espontaneamente $(66,7 \%)$, mas também trazidos por amigos $(43,5 \%)$, por algum familiar $(43,5 \%)$, encaminhados por outros serviços de saúde, Corpo de Bombeiros, delegacias de polícia e IML (41\%), identificados através de visitas domiciliares (25,6\%). Apenas um serviço mencionou a chegada de pessoas idosas por meio de denúncia.

O fluxo do atendimento ao idoso vítima de violência, na visão de um gestor de serviço préhospitalar, não difere daquele que acontece com os pacientes de maneira geral. Por outro lado, um médico afirmou que atender o paciente idoso requer "mais perspicácia" do profissional, principalmente quando se traçam as hipóteses diagnósticas, pois muitas vezes, apresentam condições de saúde mais complexas, com múltiplas doenças crônicas, uso prolongado de medicamentos, descompensam com facilidade e ainda são mais suscetíveis a infecções. Todos esses fatores fazem com que o idoso ocupe o leito por um tempo prolongado dificultando sua internação hospitalar. Somam-se a esse fato a saturação e a deficiência de vagas nos hospitais e a desarticulação entre o pré-hospitalar móvel e a recepção do paciente nas unidades de saúde do município ${ }^{10}$. Estes problemas observados no presente trabalho, e também em pesquisas anteriores, vão de encontro às orientações do Ministério da Saúde sobre a existência de uma retaguarda com serviços de referência mediados pela Central de Regulação $0^{4}$.

Um socorrista salienta que a maior demanda de sua unidade é de pacientes idosos e aponta como dificuldade no atendimento o fato de, 
Tabela 3. Distribuição da categoria e do número de profissionais segundo níveis do atendimento

\begin{tabular}{|c|c|c|c|c|}
\hline Profissionais & Pré-hospitalar & Hospitalar & Reabilitação & $\begin{array}{c}\text { Total de } \\
\text { Profissionais }\end{array}$ \\
\hline Geriatra & 11 & 9 & - & 20 \\
\hline Médico Clínico & 64 & 171 & - & 235 \\
\hline Cirurgião geral & - & 83 & - & 83 \\
\hline Médico Neurologista / Neurocirurgião & 5 & 38 & - & 41 \\
\hline Oftalmologista & 5 & 28 & - & 33 \\
\hline Cirurgião Ortopedista & - & 152 & - & 152 \\
\hline Médico Ortopedista & 3 & 227 & - & 230 \\
\hline Cirurgião vascular & - & 31 & - & 31 \\
\hline Angiologista & - & 20 & - & 20 \\
\hline Cirurgião bucomaxilofacial & - & 11 & - & 11 \\
\hline Cirurgião cardiovascular & - & 1 & - & 1 \\
\hline Cardiologista & - & 55 & - & 55 \\
\hline Hematologista & - & 29 & - & 29 \\
\hline Toxicologista & - & 22 & - & 22 \\
\hline Gastroenterologista & - & 1 & - & 1 \\
\hline Coloproctologista & - & 6 & - & 6 \\
\hline Otorrinolaringologista & - & 21 & - & 21 \\
\hline Médico/Cirurgião Ginecologista & 10 & 11 & - & 21 \\
\hline Cirurgião plástico & - & 14 & - & 14 \\
\hline Cirurgião torácico & - & 7 & - & 7 \\
\hline Anestesiologista & - & 95 & - & 95 \\
\hline Intensivista & - & 58 & - & 58 \\
\hline Radiologista & 0 & 23 & - & 23 \\
\hline Fisiatra & - & - & 6 & 6 \\
\hline Psiquiatra & 5 & 10 & 0 & 15 \\
\hline Enfermeiro & 155 & 316 & 17 & 488 \\
\hline Assistente social & 7 & 44 & 3 & 54 \\
\hline Odontólogo & 3 & 35 & - & 38 \\
\hline Psicólogo & 8 & 13 & 5 & 26 \\
\hline Nutricionista & 5 & 42 & 2 & 49 \\
\hline Fisioterapeuta & - & 122 & 6 & 128 \\
\hline Terapeuta Ocupacional & 1 & 13 & 2 & 16 \\
\hline Fonoaudiólogo & 4 & 8 & 8 & 20 \\
\hline Farmacêutico & 3 & 25 & 4 & 32 \\
\hline Técnico/auxiliar de enfermagem & 280 & 1017 & 10 & 1307 \\
\hline Agente de saúde & 6 & - & - & 6 \\
\hline Responsável técnico & - & 1 & 2 & 3 \\
\hline Auxiliar de Serviços administrativos & 16 & - & - & 16 \\
\hline Auxiliar de serviços gerais & 10 & - & - & 10 \\
\hline
\end{tabular}

- não se aplica ou não foi questão de pesquisa para esse nível do atendimento, de acordo com as Políticas.

muitas vezes, estes não conseguirem informar sobre as próprias condições de saúde. Discordando da visão do médico, afirma que o fato do paciente ser idoso não interfere na definição da internação, porque para isso usam o critério da gravidade do caso. Esse profissional reconhece que a prioridade, preconizada pelo Estatuto do Idoso, não acontece na prática. Mesmo considerando a gravidade do quadro clínico do paciente, os profissionais encontram problemas para o atendimento nas emergências que, muitas vezes, não recebem o paciente alegando falta de especialistas. Diante dessa recusa a equipe de resgate perde tempo peregrinando nos serviços em busca de atendimento ${ }^{16}$.

Quando se trata de idoso em situação de violência a conduta adotada nas unidades de socorro é o encaminhamento ao hospital, o registro dos fatos e dos sinais suspeitos na ficha/prontuário, a comunicação das suspeitas ao médico e ao assis- 
tente social da unidade. Essa comunicação acontece por iniciativa do profissional que faz o atendimento, pois, contrariando as políticas aqui consideradas ${ }^{4,5}$, não há orientações por parte da instituição sobre esse procedimento. Em muitos casos orientam a família sobre os cuidados adequados.

Para os profissionais do nível pré-hospitalar não existe uma porta de entrada diferenciada para idosos vítimas de violência nos serviços, mas durante o atendimento os casos são identificados e trabalhados com a família, com orientações, suporte e até mesmo fazendo a denúncia. No entanto, existem obstáculos que impedem uma intervenção integral, entre eles o despreparo para lidar com o tema, o número reduzido de profissionais para intervir junto às famílias e a falta de estrutura e de apoio institucional. O gestor de um serviço falou sobre sua preocupação nessa área: você faz o que com o idoso que está sofrendo uma violência? [...] é uma questão extremamente complexa que você tem que abordar, tem que atuar, dar suporte e você não tem estrutura para isso. Nesse sentido, acredita que o próprio serviço de saúde acaba assumindo o papel de agressor do idoso por não ter condições de oferecer o suporte necessário.

Quando necessita de internação hospitalar, o idoso recebe o mesmo tratamento dado aos demais usuários. Um gestor hospitalar lamenta dizendo que está longe da gente dar o primeiro atendimento diferenciado ao idoso [...], ele é mais um a entrar na fila habitual das grandes emergências. Concordando com um colega do nível pré-hospitalar reconhece que o hospital é causador de traumas para o idoso que precisa permanecer internado, pois o ambiente diferente do habitual e o afastamento da família comprometem muito seu quadro físico e emocional. Em sua fala fica claro que o direito a um acompanhante durante a hospitalização não é cumprido, o que gera desorientação, dificuldades alimentares, perda de peso, comprometimento pulmonar, imobilidade e pode chegar ao óbito sem condições de tratar a "doença primária que motivou a internação”.

Os profissionais de um hospital do município relataram uma experiência que consideram positiva e que tenta garantir a prioridade preconizada nas políticas. Ao procurar atendimento, o idoso é recebido pela enfermagem que faz uma avaliação do risco e encaminha corretamente para os setores do hospital.

Gestores e profissionais revelaram que, muito frequentemente, se deparam com o abandono da família em relação ao idoso, que mesmo com alta hospitalar não consegue voltar para casa porque nenhum familiar quer se responsabilizar por seus cuidados.

No nível de reabilitação, os profissionais foram enfáticos alegando não receberem idosos vítimas de violência, mas somente os que sofreram quedas ou possuem sequelas de doença crônico-degenerativa. Ainda relataram as dificuldades que os idosos enfrentam para seu transporte até o serviço, a interrupção do tratamento devido a incapacidades funcionais e a falta de quem os acompanhem até a reabilitação ou, quando necessário, a outro serviço.

No que se refere às atividades desenvolvidas na atenção ao idoso vítima de violência, 58,3\% dos serviços pré-hospitalares oferecem atendimento clínico, orientação à família e informações sobre direitos; em 50\% das unidades pesquisadas nesse nível acontecem atendimento individual, visitas domiciliares, ações de promoção do autocuidado, estímulo à participação em grupos e identificação da rede de suporte social do idoso. Essas ações estão em conformidade com as políticas públicas consideradas no presente trabalho.

Entre os hospitais, $40 \%$ possui equipe para a internação e assistência domiciliar de idosos e apenas $33,3 \%$ deles conta com uma equipe constituída por médico e enfermeiro, em $26,7 \%$, essa equipe tem: técnico ou auxiliar de enfermagem e geriatra. Em $20 \%$, existem profissionais com formação em gerontologia para o atendimento domiciliar. Apenas um dos serviços de reabilitação realiza a visita domiciliar, mas em nenhum deles existe uma equipe multidisciplinar para esse tipo de reabilitação de idosos vítimas de violência.

Um gestor de serviço móvel de urgência revelou que a interação da equipe com a família do idoso é papel que cabe mais ao hospital, pois sua função é o transporte do paciente. Essa visão espelha um distanciamento desse gestor com o atendimento propriamente dito e é um posicionamento contraditório ao dos profissionais. Os socorristas, mais próximos da realidade do serviço, afirmaram que a interação com a família acontece durante todo o atendimento e que os familiares são atores essenciais para o socorro do idoso. Na maioria dessas chamadas é o familiar quem faz o primeiro contato e na chegada da equipe participa ativamente do atendimento.

Fazendo referência ao conceito segundo o qual o suporte social é comparado a uma teia, um gestor indicou que muitas vezes é preciso tecer uma nova nos pontos onde há esgarçamentos dos fios. Na experiência dos profissionais, muitas famílias estão sempre presentes, mas outras 
optam em manter uma certa distância. Esse distanciamento reflete as relações conflituosas com o idoso, o estabelecimento de laços afetivos muito frouxos ao longo do tempo. Essa dificuldade enfrentada por idosos e suas famílias se constitui em fator de risco para a violência ${ }^{21}$.

Para o médico de um hospital, a família é muito bem vinda e tem dois horários para visitas diárias, que acontecem nos momentos das refeições para que possam ajudar os idosos na alimentação. Se por um lado, essa boa receptividade de familiares no ambiente hospitalar é benéfica para o idoso, também revela uma sobrecarga de trabalho para os profissionais que precisam contar com os familiares para a administração das refeições do paciente.

Na reabilitação, a presença da família parece ser mais constante, participando dos atendimentos, recebendo orientações e realizando exercícios com o paciente em casa. Também é chamada para reuniões com a equipe, acompanha o progresso do tratamento e ainda está presente nas datas comemorativas e nas festas realizadas nos serviços.

Grande parte das unidades do nível pré-hospitalar e hospitalar informou que oferece suporte e acompanhamento ao idoso vítima de violência $(62,1 \%)$ e para a família $(55,2 \%)$. Por outro lado, o apoio às equipes que prestam o atendimento às vítimas de violência é oferecido por apenas $27,6 \%$ das unidades e a atenção aos autores de violações contra os idosos é realizada por 20,7\% dos serviços estudados. Os serviços de reabilitação pesquisados não oferecem esse apoio ao idoso, à equipe e tampouco aos agressores.

No âmbito da prevenção da violência, 58,3\% dos serviços pré-hospitalares e 28,6\% dos hospitalares estudados realizam ações com esse propósito. Novamente é possível identificar uma discordância com as normas estabelecidas pelas políticas no que diz respeito à prevenção da violência. Deslandes et al. ${ }^{17}$ também identificaram a escassez de ações dessa natureza na atenção às vítimas de violência no Brasil, no entanto, no município do Rio de Janeiro, 64\% dos serviços analisados por esses autores informaram realizar prevenção da violência em geral, sem as especificidades da pessoa idosa. Considerando o grupo dos idosos, a PNRMAV e a PNSPI destacam a importância dessas ações para a promoção da qualidade de vida e não apenas o cuidado tradicional às consequências da violência.

Pequeno percentual dos serviços pesquisados nos três níveis $(34,5 \%)$ realiza notificação sistemática dos casos suspeitos ou confirmados de violência contra o idoso, como preconiza o Estatuto. Apenas 20,7\% possuem ficha para essa notificação, outros 48,3\% informaram que a notificação dos casos depende do profissional que faz o atendimento e 10,3\% não a realizam. Nenhum dos serviços de reabilitação estudados faz a notificações para outras instituições.

Muitos estudos têm apontado as barreiras encontradas pelos profissionais de saúde na realização da notificação. Apesar de ser uma diretriz da PNRMAV, do Estatuto do Idoso e de estar contemplada nos códigos de ética de algumas profissões como medicina, enfermagem, odontologia e psicologia ${ }^{22}$, ainda não é uma prática tão naturalizada nos serviços, como observado no município do Rio de Janeiro. Entre as barreiras para a notificação dos casos encontram-se: a dificuldade em identificar a violência e a pouca familiaridade dos técnicos com procedimentos de notificação ${ }^{23}$; a percepção de que notificar não é papel do profissional de saúde, que deve se preocupar com a situação clínica do paciente ${ }^{24}$; a visão de que o ato de notificar é uma medida punitiva e o medo de represálias por parte do possível agressor ${ }^{25}$.

Nas entrevistas, os profissionais do atendimento pré-hospitalar lamentaram a difícil interlocução com a rede de proteção ao idoso no município. Informaram que esse contato é informal e que os idosos passam por atendimentos morosos e pouco eficientes. Os do nível hospitalar revelaram que não têm contato com a rede e os da reabilitação nem mesmo fizeram referência a ela, indicando a fragilidade das articulações entre os serviços. Os depoimentos revelam que esses serviços existem e que trabalham isoladamente, dando pistas da necessidade da construção do fluxo entre eles e de uma rede de atenção e proteção que atue de forma integrada no município. Essa integração é essencial no atendimento às vítimas de violência, fato que também é reforçado nas diretrizes da PNRMAV. Essa desarticulação para o atendimento às pessoas vitimizadas pela violência no Rio de Janeiro já foi apontada em estudos anteriores como motivo de crítica dos gestores do setor saúde ${ }^{17}$.

Solicitados a avaliar o atendimento prestado aos idosos vítimas de violência, os profissionais do pré-hospitalar afirmam que a atenção prestada em seu nível consegue atingir os objetivos propostos pelo serviço, dá prioridade aos idosos, trabalha para diminuir as comorbidades e o tempo de internação do paciente. Outros reconhecem que os idosos vítimas de violência encontram muitas dificuldades como a pouca capacitação dos pro- 
fissionais para identificar e lidar com esses casos. Um gestor afirmou que poucos serviços têm condições de realizar esse atendimento, acionar a rede de suporte e orientar a família.

No nível hospitalar, os profissionais acreditam que o atendimento oferecido está evoluindo na medida do possível. Visão contrária a anterior tem os que trabalham na reabilitação, revelando que o idoso vítima de violência é mal atendido nos serviços de saúde, principalmente nos hospitais.

\section{Considerações finais}

O presente estudo apresenta algumas limitações como a natureza local das informações e a abordagem não probabilística da amostra. A natureza local dos dados não possibilita extrapolações para outros municípios, mas retrata uma realidade semelhante à observada em outras redes no país ${ }^{10}$. A pesquisa não visou a abordagem de uma amostra probabilística de serviços, mas sim uma aproximação da realidade a partir de uma amostra de conveniência (indicada pelos gestores da área). Esta opção metodológica se deu em função do pouco conhecimento sobre a rede de serviços em relação à especificidade do atendimento à pessoa idosa em situação de violência. Nesse sentido, a visibilidade das adequações necessárias para que o setor saúde responda à emergência e a importância do tema em questão permitem conhecer os desafios e as possibilidades que se impõem na construção da integralidade do atendimento.

A implantação e a implementação das políticas aqui consideradas se dá de forma muito diferenciada entre as unidades pesquisadas, um pou- co mais nas de nível pré-hospitalar e hospitalar e muito aquém do desejado no atendimento de reabilitação.

As lacunas identificadas na reabilitação de idosos vítimas de violência revelam uma atenção muito focada na clínica ortopédica e neurológica do paciente, contrariando os esforços das políticas e das orientações do Ministério da Saúde que preconizam a atenção integral em todos os níveis.

A abordagem a questão da violência nos serviços de saúde e, principalmente, com uma pessoa idosa requer sensibilização para o tema, capacitação para a atuação e articulação intrassetorial e com a rede de proteção. Este é um desafio aos gestores do setor saúde do município do Rio de Janeiro para os três níveis de complexidade do atendimento.

Além disso, algumas questões vêm a tona, a partir das análises realizadas, como a necessidade de incluir o tema violência nos atendimentos dispensados ao idoso, pois muitas vezes o mesmo não verbaliza o abuso e não apresenta sinais claros de sua presença; proporcionar maior visibilidade às especificidades da atenção à mulher idosa na rede de serviços de saúde; apoiar, orientar e instrumentalizar as famílias para o cuidado necessário, tentando criar estratégias para o fortalecimento e o resgate dos laços afetivos e assim evitar o abandono em instituições hospitalares; investir na vigilância das alterações que levam a incapacidades funcionais; ampliar as ações de prevenção da violência e promoção da saúde e da qualidade de vida do idoso, como preconizado nas políticas públicas.

Espera-se que a partir de estudos como este seja possível visualizar os desafios e planejar estratégias para a garantia de uma atenção especializada e eficiente às pessoas idosas vítimas de violência no país.

\section{Colaboradores}

AP Ribeiro e ER Souza trabalharam na concepção, análise, escrita e revisão final do artigo e FC Valadares na escrita e revisão final.

\section{Agradecimento}

Ao $\mathrm{CNPq}$ e à Fundação Carlos Chagas de Amparo à Pesquisa do Estado do Rio de Janeiro. 


\section{Referências}

1. Minayo MCS, Souza ER. Violência contra idosos: é possível prevenir. In: Brasil. Impactos da violência na saúde dos brasileiros. Brasília: Ministério da Saúde, Secretaria de Vigilância em Saúde; 2005.

2. Brasil. Ministério da Saúde. Datasus. Sistema de Informação sobre Mortalidade, 2010. [site na Internet]. [acessado 2010 fev 24]. Disponível em: http:// www2.datasus.gov.br/DATASUS/index.php?area= 0205

3. Brasil. Ministério da Saúde. Datasus. Sistema de Informações Hospitalares do SUS - SIH/SUS, 2010. [site na Internet]. [acessado 2010 fev 24]. Disponível em http://www2.datasus.gov.br/DATASUS/index. php?area $=0203$

4. Brasil. Portaria MS/GM no 737 de 16 de maio de 2001: Política Nacional de Redução da Morbimortalidade por Acidentes e Violências. Diário Oficial da União 2001; 18 maio.

5. Brasil. Lei no 10.741 de $1^{\circ}$ de outubro de 2003. Estatuto do idoso. Diário Oficial da União 2003; 3 out.

6. Brasil. Portaria no 2528, de 18 de outubro de 2006: Política Nacional de Saúde da Pessoa Idosa. Diário Oficial da União 2006; 19 out.

7. Camarano AA, Pasinato MT. O envelhecimento populacional na agenda das políticas públicas. In: Camarano AA, Pasinato MT, organizadores. Os novos idosos brasileiros: muito além dos 60 ? Rio de Janeiro: Ipea; 2004. p. 253-92.

8. Minayo MCS, Assis SG, Souza ER. Avaliação por triangulação de Métodos: abordagem de programas sociais. Rio de Janeiro: Ed. Fiocruz; 2005.

9. Brasil. Ministério da Saúde. Datasus. Estimativas populacionais, 2010. [site na Internet]. [acessado 2010 set 13]. Disponível em: http://tabnet.datasus.gov.br/ cgi/tabcgi.exe?ibge/cnv/popRJ.def

10. Minayo MCS, Deslandes SF. Análise diagnóstica da política nacional de saúde para redução de acidentes e violências. Rio de Janeiro: Ed. Fiocruz; 2007.

11. Souza ER, Ribeiro AP, Atie S, Souza AC, Marques CC. Rede de proteção aos idosos do Rio de Janeiro: um direito a ser conquistado. Cien Saude Public 2008; 13(4):1153-1164.

12. Bardin L. Análise de conteúdo. Lisboa: Edições 70; 1979.

13. Minayo MCS. O desafio do conhecimento. São Paulo: Hucitec; 2006.

14. Brasil. Ministério da Saúde. Datasus. Cadastro Nacional dos Estabelecimentos de Saúde do Brasil - CNES, 2010. [site na Internet]. [acessado $2010 \mathrm{fev}$ 12]. Disponível em: http://www2.datasus.gov.br/ DATASUS/index.php?area $=0204$

15. Albuquerque VS. Violência sob o olhar e o agir de quem socorre: representações dos profissionais do atendimento pré-hospitalar de urgência e emergência [tese]. Rio de Janeiro: Escola Nacional de Saúde Pública; 2010.
16. Brasil. Portaria GM, 2.048, 5 nov. 2002. In: Brasil. Política Nacional de Atenção às Urgências. Brasília: Ministério da Saúde; 2004.

17. Deslandes SF, Souza ER, Minayo MCS, Costa CRBSF, Krempel M, Cavalcanti ML, Lima MLC, Moysés SJ, Leal ML, Carmo CN. Caracterização diagnóstica dos serviços que atendem vítimas de acidentes e violências em cinco capitais brasileiras. Cien Saude Public 2006; 11(Supl.):1279-1290.

18. Instituto de Pesquisa Econômica Aplicada (IPEA), Departamento Nacional de Trânsito (DENATRAN), Associação Nacional de Transporte Público (ANTP). Impactos sociais e econômicos dos acidentes de trânsito nas rodovias brasileiras. Relatório executivo. Brasília: Instituto de Pesquisa Econômica Aplicada (IPEA), Departamento Nacional de Trânsito (DENATRAN), Associação Nacional de Transporte Público (ANTP); 2006.

19. Vieira EM, Perdona GCS, Almeida AM, Nakano AMS, Santos MA, Daltoso D. Conhecimento e atitudes dos profissionais de saúde em relação à violência de gênero. Rev. bras. Epidemiol 2009; 12(4):566-777.

20. Deslandes SF, Minayo MCS, Lima MLC. Atendimento de emergência às vítimas de acidentes e violências no Brasil. Rev Panam Salud Publica 2008; 24(6):430-440.

21. Souza ER, Ribeiro AP, Penna LHG, Ferreira AL, Santos NC, Tavares CMM. O tema violência intrafamiliar na concepção dos formadores dos profissionais de saúde. Cien Saude Public 2009; 14(5):1709-19.

22. Saliba O, Garbin CAS, Garbin AJI, Dossi AP. Responsabilidade do profissional de saúde sobre a notificação de casos de violência doméstica. Rev Saude Publica 2007; 41(3):472-477.

23. Taylor DK, Bachuwa G, Evans J, Jackson-Johnson V. Assessing Barriers to the identification of elder abuse and neglect: a communitywide survey of primary care physicians. J Natl Med Assoc. 2006; 98(3):403-404.

24. Tilden VP, Schmidt TA, Limandri BJ, Chiodo GT, Garland MJ, Loveless PA. Factors that influence clinicians' assessment and management of family violence. Am J Public Health 1994; 84(4):628-633.

25. Halphen JM, Varas GM, Sadowsky JM. Recognizing and reporting elder abuse and neglect. Geriatrics 2009; 64(7):13-18.

Artigo apresentado em 16/09/2010

Aprovado em 24/11/2010

Versão final apresentada em 30/11/2010 